\title{
Innovative Talent Education in the United States and Its Enlightenment to China-Take the TI: GER Project as an Example
}

\author{
Shu Dong \\ School of Economics and Management, Changshu Institute of Technology, Suzhou, China \\ Email address: \\ Jdp129@126.com \\ To cite this article: \\ Shu Dong. Innovative Talent Education in the United States and Its Enlightenment to China-Take the TI: GER Project as an Example. Science \\ Discovery. Vol. 6, No. 5, 2018, pp. 338-343. doi: 10.11648/j.sd.20180605.15
}

Received: August 13, 2018; Accepted: September 15, 2018; Published: October 18, 2018

\begin{abstract}
Professor Marie C. Thursby found that because of the difficulty of effectively transmitting hidden knowledge in the process of commercialization and the shortcomings of the existing innovation system in the United States, the probability of success from technological innovation to commercialization is low in the United States. Therefore, she insisted on that the Science \& engineering universities of US must carry on innovative talent education in order to enhance the competitiveness of American manufacturing by affording their mater of multidisciplinary knowledge and know of commercialization process after her study on American's innovation and it's commercialization. Professor Marie C. Thursby had put forward the TI: GER program based on student education perspective, and this program has achieved a good result. China wants to develop from a large manufacturing country to a powerful manufacturing country, it is necessary to strengthen the construction of innovative talents. Learning from the advanced talent training model of the United States will help Chinese universities to improve their talent training methods and strengthen the innovative talents' training. In this paper, case study is used to analyze the background, project content and implementation effect of TI: GER in detail. The conclusion of the paper is that China should improve the current innovative talent training mode from three aspects: market demand-oriented professional division of labor, project-driven flexible teaching management and reform of the existing assessment mechanism of university teachers.
\end{abstract}

Keywords: America, Innovative Talent Education, TI: GER

\section{美国创新型人才教育及其对中国的启示一以佐治亚理工学院 TI:GER项目为例}

\author{
董舒 \\ 常熟理工学院, 经济与管理学院, 苏州, 中国 \\ 邮箱 \\ Jdp129@126.com
}

摘要: 玛芮·瑟斯比教授在对美国技术创新和商业化的研究中发现, 由于在商业化过程中隐形知识难以得到有效的传递 和美国现有创新制度的不足，导致美国从技术创新到商业化成功的概率较低。为此，她提出为了提升美国制造业的竞 争力, 为其提供掌握多学科知识、又了解市场商业化过程的人才, 在美国的工科院校, 必须进行创新型教育, 她从人 才教育的视角提出了 TI: GER综合培养研究项目, 并取得了良好的效果。中国要从一个制造业大国发展为一个制造业 强国, 加强创新型人才队伍的建设必不可少, 对美国先进的人才培养模式进行学习, 有助于中国高校改进人才培养方 式、加强创新型人才培养。本文采用案例研究的方法, 详细分析了 TI: GER的产生背景、项目内容及其实施效果。文 
章结论认为, 中国应该从以市场需求为导向的专业分工、以项目驱动的柔性教学管理和改革现有的高校教师的考核机 制三个方面改进现有的创新型人才培养模式。

关键词：美国，创新型人才教育，TI：GER

\section{1. 引言}

美国是全球创新的领导者，长期以来，美国的创新指 数位居世界第一。在美国强大的创新能力背后，美国高校 对学生创新能力的培养功不可没。在美国一百多年的创新 实践中, 美国的高校非常重视对学生创新能力的培养, 并 且形成了一套行之有效的创新型人才的培养模式。其中, 最为典型的是哈佛大学的“案例教学”模式和斯坦福大学 的“校企合作”模式。上述两种模式已被广为研究, 成为高 校人才培养的典范。美国知名学者、佐治亚理工学院商学 院战略管理教授玛芮·瑟斯比（Marie C. Thursby）在长期 对美国创新、创业和商业化进行研究和实践的基础上，提 出了“技术创新-创造经济价值”（TI：GER）的人才培养模 式。该项目自2002年实施以来, 已获得了美国国家科学基 金会等机构高达 1400 多万美元的资金资助, 培养的研究生 在创新和创业领域取得了显著的成效。玛莴·瑟斯比教授 也因TI: GER项目的发起者及主持人成为全球创新型人才 教育的知名学者, 并于 2014年多次受邀在中国知名大学就 TI: GER项目的运行与实施进行了交流*。

中国在“十二五”提出了国家自主创新能力建设的规 划, 其中重要内容之一是要加强创新型人才队伍的建设。 高校作为培养高素质人才的基地, 长期以来, 中国高校更 多地把教育的重点放在知识的传授上，但在创新能力、实 践能力上却满足不了社会对高质量人才的需求 [1]。因此, 对美国先进的人才培养模式进行学习, 有助于中国高校改 进人才培养方式、加强创新型人才培养。本文在分析了 TI: GER项目产生背景的基础上, 介绍了该项目的主要内容与 特点, 并结合中国当前高校的人才培养模式, 提出了管理 启示。

\section{TI：GER项目的产生背景}

TI：GER全称“技术创新-创造经济价值”（TI：GER Technological Innovation: Generating Economic Results）。 该模式是将不同专业类型的研究生整合成为一个研究小 组, 旨在为学生提供多学科知识教育的一种人才培养模式。 玛苪·瑟斯比教授在长期对美国技术创新与商业化过程的 研究中发现, 即使在整个创新系统较为完善的美国, 一项 技术创新从实验成功到商业化应用的成功概率仍然很低 $[2 ， 3]$ 。瑟斯比教授认为，导致美国技术创新商业化应用 率低的主要原因有下述两点。

* 参见上海财经大学 (上海, 中国) 、中国人民大学 (北京, 中国) 以 及电子科技大学 (成都, 中国) 等网站相关报道。

\section{1. 隐性知识的重要性}

隐性知识是指人们知道, 但难以用语言表达的部分。 相比较显性知识而言, 隐性知识具有独占性、难以规范性、 不易转移性等特征。瑟斯比教授研究发现, 在技术创新领 域, 隐性知识扮演着重要的角色。她在比较了美国高校和 企业的技术创新中研究发现，在技术创新的各阶段中，尤 其在创新的前期阶段, 隐性知识起着非常重要的作用。如 表1所示。

表1 隐性知识在技术创新各阶段所占的百分比。

\begin{tabular}{lll}
\hline 阶段 & 高校 (\%) & 企业 $(\%)$ \\
\hline 概念形成 & 45 & 38 \\
形成原型 & 37 & 36 \\
前期实验 & 26 & 15 \\
正式实验 & 10 & 5 \\
市场化可行性研究 & 15 & 9 \\
商业化应用 & 12 & 7 \\
\hline
\end{tabular}

(数据来源[4]: Dechenaux \& Thursby, 2011)。

在商业化的过程中, 由于隐性知识难以得到有效的 转移, 导致技术创新在商业化应用的过程中存在较高失 败率。这是因为, 第一, 在美国技术创新到商业化应用 的过程中, 研发人员在创新的各个阶段投入的精力不同。 在概念实证和原型实验阶段, 研究人员投入的精力的百 分比分别是 $38 \%$ 和 $36 \%$, 而在市场化可行性研究和商业化 阶段分别是 $9 \%$ 和 $7 \%[5]$ 。第二，在上述条件下，尤其是 具有专业知识的高校教师，在前两个参与的比重分别为 $55 \%$ 和 $54 \%$, 而在后两个阶段分别为 $15 \%$ [5]。正是由于这 些专业技术人员在市场化阶段投入的精力和参与的比例 较低, 导致在商业化应用过程中, 隐性知识难以有效的 得到转移, 造成了较高的失败率。瑟斯比教授在对创新 失败的案例研究中发现, 除了在形成原型和正式实验两 个阶段具有较高的失败率 (两项和 $72 \%$ ) 之外, $43 \%$ 失败 来自于商业化应用阶段。

\section{2. 美国现有创新制度的不足}

美国的国家创新体系主要由四个部分组成: 政府、高 校、企业和非盈利组织。其中, 政府通过规划和立法对国 家创新体系起引导作用, 高校主要负责进行基础研究和人 才教育, 企业主要负责进行技术创新, 非盈利组织是对上 述三者的一个有益补充。这四个部分分工明确, 又相互协 作, 形成了一个较为完善的国家创新体系。美国政府一方 面通过高额的科研经费预算, 加大对基础科研的投入, 尤 其是奥巴马入主白宫以来，将美国GDP的3\%投入到研究 和创新领域，成倍的增加美国国家科学基金会（NSF）等 国家主要科研机构的经费[6]; 另一方面, 又通过制定专利 保护体制、规范市场竞争等一系列措施, 推动政府、企业、 
高校和研究机构加强科研合作, 提高科研成果的转化率。 在这一体系下, 美国成为了拥有着全球最多创新成果和高 科技人才的国家。

表2 技术创新的商业化应用的失败率及其原因。

\begin{tabular}{ll}
\hline 百分比 & 失败原因 \\
\hline 46.5 & 技术失败 \\
26.3 & 滞后于市场时间太长 \\
17.6 & 缺乏科研人员的帮助 \\
12.6 & 技术侵犯了其他知识产权 \\
11.1 & 其他因素 \\
\hline
\end{tabular}

（资料来源[5]：Thursby，2007）

然而, 即使在拥有较为完善的国家创新体系的美国, 由于科研院校和企业在创新的动机与目标上的不同, 导致 一项创新从概念原型的形成到商业化应用失败率较高。科 研院校的创新是以技术为导向, 高校教师的研究兴趣在于 理论研究, 研究目标在于取得理论上的突破与进展。然而, 企业是以利润为导向, 注重的是一项创新的商业应用及市 场前景。高校教师具有专业知识, 但是缺乏对技术应用的 市场化方面的知识; 而企业了解技术应用市场化方面的知 识, 但缺乏技术创新方面专业的知识。这就导致了许多高 校的技术创新在商业化应的转化率低。例如, 按照美国现 行的技术转让条例, 一项研究在实验室成功之后, 研究人 员认为该项技术具有一定的市场前景的条件下想要寻求 技术转让, 必须向当地技术转让许可办公室提出申请, 获 得许可 (Licensing)。因为在美国, 高校的科学研究是受 到政府资助的, 因此在提交技术转让的过程中, 必须要获 得政府的许可。在获得许可之后, 研究人员就向技术转让 办公室提交 (Boarding) 该项目研究的相关信息, 寻求企 业的合作。但瑟斯比教授对美国11个主要的研究性大学的 4998名科研人员在1983-1999年间技术创新的研究发现, 在 1983 年只有 $2.7 \%$ 的科研人员提交了他们的申请, 而在 90 年代这一数字也仅停留在 $10 \%-11 \%$, 然而, 有 $63.5 \%$ 的科 研人员从未对他们研究项目提出过申请, $14.6 \%$ 的人员对 他们的研究项目提出申请仅一年之后由于没有获得企业 的技术认领而撤销了该申请 [5]。究其原因在于, 许多科学 研究项目在实验室取得了成功, 但由研究人员不了解该项 研究的市场状况没有进行技术转让申请, 或在技术的应用 方面以及成本等方面的问题难以进行商业化应用。
通过对美国技术创新到商业化应用存在较高失败率 的研究, 瑟斯比教授意识到, 提升美国从技术创新到市场 化应用的转化率, 要从根本上改变现有的技术转让模式, 一方面要让研发人员了解市场以及企业运行规律, 另一方 面, 要让企业管理人员熟知技术研发的过程。从高校人才 培养的视角来看, 为了将来能够给美国制造业的技术创新 与商业化应用 (根据美国国家科学院科技和公共政策委员 会的统计, 在美国每年超过 $50 \%$ 的理工科毕业生进入到了 工业企业就业）提供掌握多学科知识、又了解市场商业化 过程的人才, 在美国的工科院校, 必须进行创新型教育。 因为学生是当前和未来的发明家, 学生需要理解商业化的 基本原理, 以便能够将来提升美国制造业的竞争力。在此 背景下, TI: GER项目应用而生。

\section{TI：GER项目内容介绍}

TI: GER项目是在佐治亚理工学院和埃默里大学培养 基于具有技术创新和企业管理能力的一个综合性的研究 项目, 该项目成立于2002年, 由美国国家科学基金会出资 资助进行的综合教育和研究培训项目。该项目自2002年实 施以来, 已培养了近 400 名不同专业的学生。

\subsection{TI: GER项目介绍}

TI: GER是一项基于团队的教育项目，它是通过把博 士研究生的技术创新经过商业化的应用, 使整个团队成员 在一起互相学习, 以弥补传统教育中由于学科分类而导致 学生综合能力不足的问题。因此, 它在人员构成、课程设 置和教学方式上面和传统教育具有差别。

（1）人员构成

TI: GER的一个学生团队由不同学科的 5 名学生组成: 1 名为科学与工程专业方面的博士研究生, 2 名工商管理硕 士在读研究生, 分别由市场营销和财务管理专业的学生组 成, 另外 2 名是法学博士研究生, 分别由专利法和科技法 方面的学生组成。TI: GER的教学团队除了担任各门课程 的导师外, 还有来自于法律、商业和工程技术方面的校外 专家组成, 并由这些导师组建TI: GER项目董事会, 负责 整个项目的整体运行情况。这些团队成员每学期至少要和 各自负责的学生团队开2次会议。

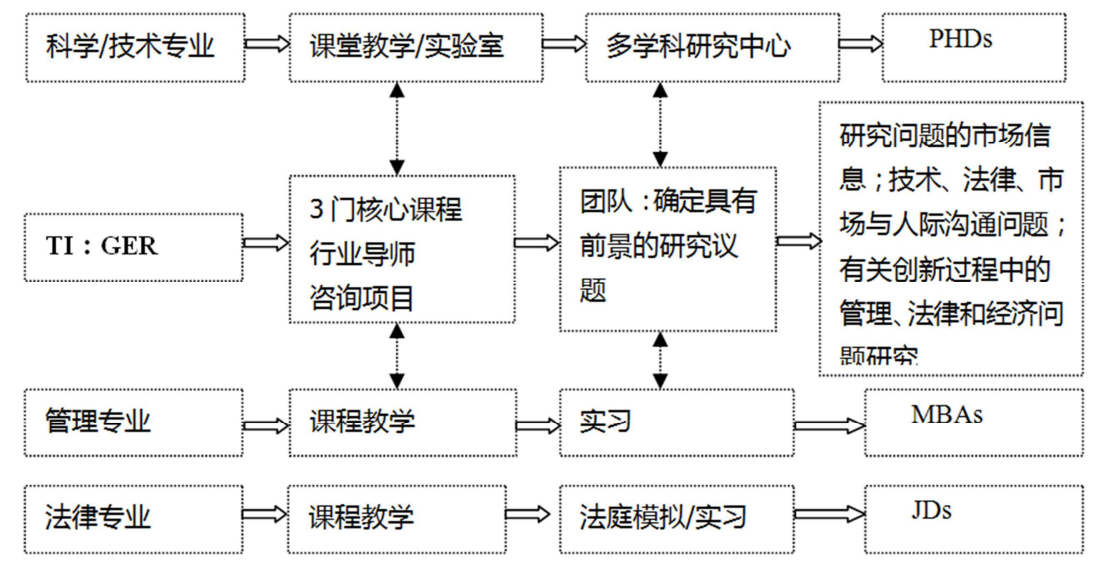

图1 TI: GER项目教育的整体思路图（资料来源：作者整理）。 


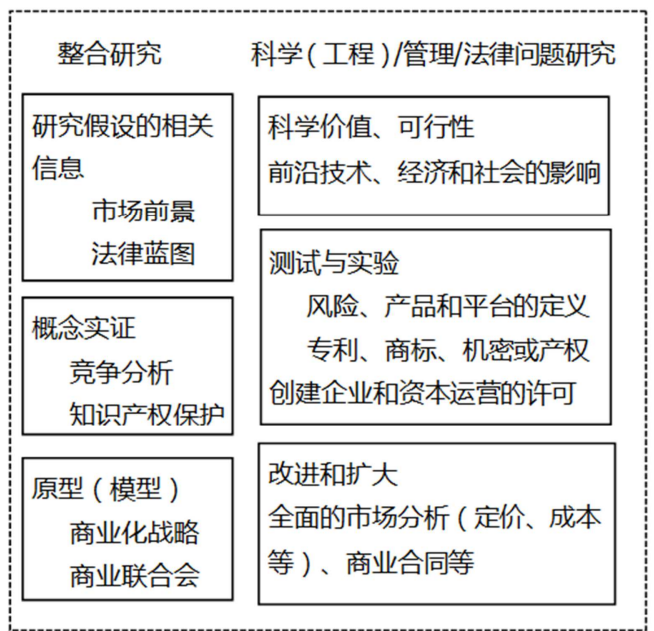

图2 TI: GER成员独特的团队体验（作者整理）。
另外, 在招收学生的过程中, 要对学生进行一项综合 测评, 主要评估他们的团队协作能力、抗压能力以及独立 完成任务的能力, 通过评估的学生才能进入TI: GER团队, 并在一起学习完团队手册和完成一些活动（练习）之后, 根据自己的研究兴趣组合成一个研究小组。

(2) 课程设置

TI: GER是要将博士研究生的技术创新进行商业化的 应用, 因此, 主要针对三个不同专业的学生专业特点, 围 绕TI: GER项目的运行来进行课程设置。主要思路是通过 项目的运行来整合三个不同专业教学与管理, 并通过考核 授予他们相应的学位。项目教育的整体思路如图1所示。

在具体的课程设置方面, TI: GER一个项目的整体运 行时间为 2 年, 每年分为春秋两个学期, 共四个学期。课 程共有 11 门核心课程和 2 门选修课程, 分为创新基础和专 题研究两个部分。课程的具体设置如表3所示。

表3 TI: GER课程安排。

\begin{tabular}{llll}
\hline 春季 & 秋季 & 春季 & 秋季 \\
\hline 创新基础 $(\mathrm{I})$ & 创新基础 $(\mathrm{II})$ & 专题 (I) & 专题 (II) (选修) \\
团队建设与发展 & 营销战略 & 项目规划与管理 & 商业计划竞赛 \\
知识产权分析 & 公司估值 & 商品化计划与拓展 & 海外合作项目 \\
行业分析 & 融资战略 & 商业计划与发展 & 其他 \\
\hline
\end{tabular}

（资料来源：作者整理）

\subsection{TI: GER项目特点}

从TI: GER项目的运行来看, 它实质上是一项整合教 育研究项目, 它贯穿了在技术创新到商业化过程中所需的 知识技能, 打破了传统教育中根据学科分类进行教育的边 界, 它是基于市场需求为导向的任务解决型的综合型人才 培养模式, 而非基于传统的专业分工的知识教育模式。TI: GER成员从一项技术创新入手, 全程参与了从技术创新到 其商业化过程的各个阶段, 以及在各阶段可能面临的问题 及其解决方案。因此, TI: GER成员不仅在项目运行过程 中学习到了从技术创新到其商业化过程中所需的知识技 能, 更重要的是成员之间通过相互协作, 共同努力, 使得 不同专业背景的成员能够感知到对方思考问题的思维方
式, 这在一定程度上有效的解决了从技术创新到商业化过 程中隐性知识转移的问题。

\subsection{TI: GER教学效果}

TI：GER项目自2002年实施以来，目前已培养了 400 多名研究生, 所培养的学生主要进入了制造业 (如宝马公 司）和咨询服务业（如DELTA）就业，并且已成长为全 球产品经理和全球客户经理等重要岗位的关键领导, 还有 许多学生创立了自己的公司。此外, 在对参与了 TI: GER 项目的学员和非TI: GER项目的学生进行横向比较研究后 发现, 参与了 TI: GER项目的学员在对技术商业化过程中 所需知识的掌握、对一项技术市场价值的理解等 9 项知识 技能方面比没有参与 TI: GER项目的学生有着显著的提升。 如图3所示。

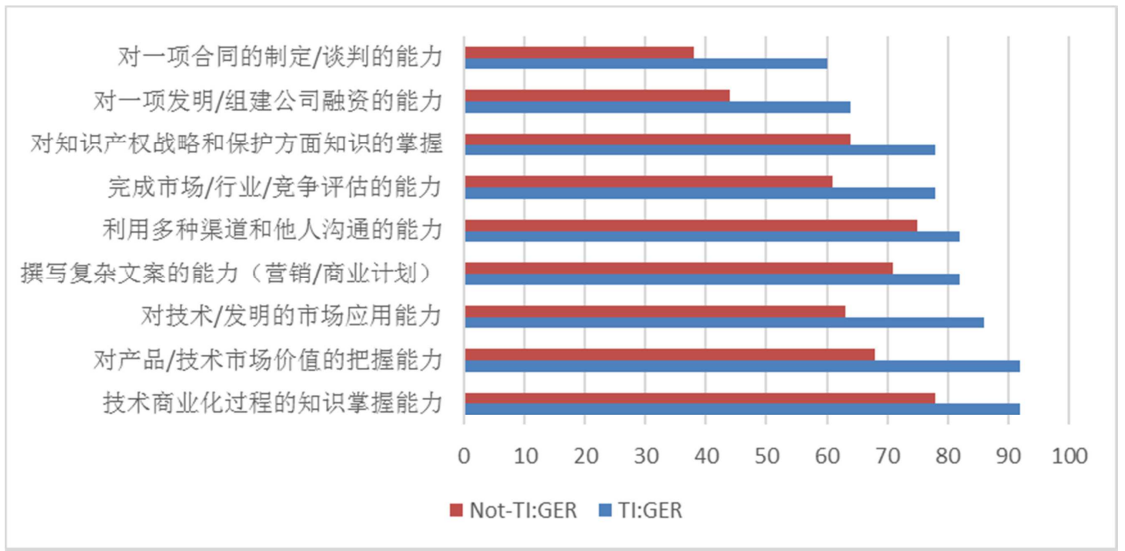

图3 TI: GER成员与非TI: GER成员的知识技能比较（资料来源：作者整理）。 


\section{4. 管理启示}

受传统教育模式的影响, 中国高校把教育的重点放在 了知识与技能的传授方面, 但对学生创新思维与能力的培 养不足[7]。尽管自2007年以来, 中国高校实施了“高等学 校教学质量与教学改革工程”, 大学在提升教育水平, 实 施创新教育方面取得了一定的成果。例如, 电子科技大学 开展了“信息技术专业素质和经济管理才能兼备的复合型 未来领军人才”为目标创新教育实验班。但从整体上来看, 中国高校的创新教育还游离于整个专业教育的体系之外 [8], 对创新教育也没有形成一个比较完整的界定、实施方 案和考核体系 [9]。尽管中美两国的国情、教育发展的水平 不尽相同, 但笔者认为, 从 TI: GER项目的产生过程和实 施情况来看, 对中国高校的创新教育具有以下几点启示。

\section{1. 实施从专业分工向市场需求为导向的专业课程设置 改革}

传统的教育模式是以学科的专业分工为导向进行的 课程设置, 这样设置的优点是学生能够系统掌握本专业的 知识体系, 对本专业有着较深的领悟力和理解力, 培养的 是学生的一种专业思维。这种专业是建立在社会能够精细 分工、紧密协作的基础之上。但从瑟斯比教授对美国技术 创新到商业化实施的研究来看, 这种分工不利于隐性知识 的转移, 难以培养具备多学科知识、解决社会现实需要的 这一“类”的人才需求。例如, 中国高校的市场营销专业, 很少涉及产品开发与大众传播方面的课程, 而企业恰恰需 要的是既懂得产品开发、又能够与大众媒体进行沟通的这 一类的市场营销人才。因此, 要对中国现行的专业课程的 设置进行改革, 培养以市场需求为导向的对人才 “类”的需 求, 而非是某一专业的需求。

\section{2. 打破现有教育按专业班级进行教育管理的模式, 实 施以项目驱动的柔性教学管理模式。}

中国现有的按专业班级进行教育管理的高校管理模 式, 便于集中授课、统一管理。但不足在于, 学生面对的 是教材和教师, 在以学习成绩为主的综合测评中, 学生考 虑的是“我”如何更好的学习, 面对的是一项客观对象, 思 维方式以我为主。况且, 这种教育方式容易让学生产生为 什么而学的疑问, 学习的动力和目标不清晰 [10]。实施以 项目驱动为主的柔性教育管理模式, 首先, 学生的学习目 标明确。在该项目中, 学生自己扮演的角色以及承担的任 务非常清晰, 这样会使学生具有明确的学习目标和动力。 其次, 在项目团队中, 学生是通过与他人进行合作来完成 一项任务。这样会使学生更多的进行横向参考、互相学习, 有助于提升学生的综合素养。第三, 以项目驱动的教学管 理, 能够使学生对项目的整体运行情况全面了解, 有助于 培养社会对高素质人才需求的满足。

\section{3. 改革现有的高校绩效考核机制, 从科研加工作量的 考核机制逐渐转向人才培养的考核机制}

现有的高校教师的考核机制主要以完成科研和教学 的任务量为主。在这种考核机制下, 教师只重视自己的科 研成果和常规教学, 而忽视了学生的就业状况和市场对人 才的需求情况，教师只对“上”负责，而不是对“下”负责。 这种机制造成的负面结果是高校教育与市场需求脱离, 难 以培养出市场需要的高素质人才, 导致大学生毕业后就业 困难, 带来种种社会问题。因此, 应该改革现有的高校教 师考核制度, 从短期的科研任务和教学任务为主的考核体 系逐渐向长期对人才培养的社会贡献指数的考核体系转 变。这就需要进一步完善现有的考核制度和社会对人才评 价的体系。

\section{5. 结论}

创新型人才的培养已是当前各个国家关注的重点内 容之一。本文详尽的介绍了 TI: Ger项目的产生背景、项 目内容与实施效果。从TI: Ger项目的成功我们可以得出, 中国高校要对创新型人才培养模式进行改革, 一是要实施 从专业分工向市场需求为导向的专业课程设置的改革; 二 是要打破现有教育按专业班级进行教育管理的模式, 实施 以项目驱动的柔性教学管理模式; 三是要改革现有的高校 绩效考核机制, 从科研加工作量的考核机制逐渐转向人才 培养的考核机制。

\section{基金项目}

江西省教育改革课题（JXJG-14-16-15）。

\section{参考文献}

[1] 王志明.职业院校工程创新人才培养策略研究[J].国内高等 教育教学研究动态,2018 (3):11-11。

[2] Rector A M, Thursby M C. Licensing inventions from entrepreneurial universities: The context of BayhDole[M]//Technological innovation: Generating economic results. Emerald Group Publishing Limited, 2016: 361-413.

[3] Thursby J G, Haeussler C, Thursby M C, et al. Prepublication disclosure of scientific results: Norms, competition, and commercial orientation[J]. Science advances, 2018, 4(5): eaar2133.

[4] Dechenaux E, Thursby J, Thursby M. Inventor moral hazard in university licensing: The role of contracts[J]. Research Policy, 2011, 40(1): 94-104.

[5] Thursby J, Thursby M. University licensing[J]. Oxford Review of Economic Policy, 2007, 23(4): 620-639.

[6] 孙昭钸.美国政府引导建立科技创新体系的主要举措[J].当 代世界,2010(5): 65-66。 
[7] 刘德才.创新思维视角下高校辅导员职业发展研究[J]. 学校 党建与思想教育:上, 2018(3): 78-80。

作者简介

[8] 张跃飞,陈劲光.关于高校创新教育策略的研究 [J].教育探索, 2014 (2): 87-88。

[9] 陈翠荣.大学创新教育实施困境的博弯分析 [J].中国高教研 究, 2014 (7): 81-84。

[10] 赵群,卢琳,任婷婷. 新工科背景下创新教育管理方式探索 [J]. 创新与创业教育,2018,9(01):65-68。

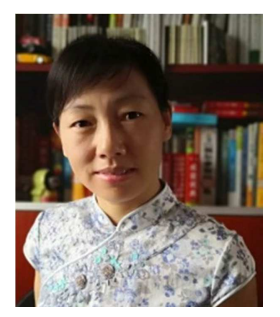

董舒（1976-），女，副教授，企业管 理专业硕士，研究方向为财务管理。 